\title{
La planificación, un instrumento para consolidar el desarrollo local en el caso de la región tres del Ecuador.
}

\begin{abstract}
(c) (i) (ㅇ)
Planning, an instrument to consolidate local development in the case of region three of Ecuador.
\end{abstract}

Ray Jonathan Aragón León. ${ }^{1}$, Dorian Damián Flores Aguilera. ${ }^{2}$ \& Yonimiler Castillo Ortega. ${ }^{3}$

Recibido: 07-01-2021 / Revisado: 12-01-2021 /Aceptado: 06-02-2021/ Publicado: 05-03-2021

\begin{abstract}
DOI: https://doi.org/10.33262/concienciadigital.v4i1.2.1580
\end{abstract}
Introduction: In the framework of these previous models and the experience acquired, Ecuador promoted, starting in 2008, the implementation of planning as a system, based on a development regime, which determined territorial management and development plans by levels of government, aimed at resolving territorial imbalances that promote development and socio-economic-environmental equity of the populations. Objective: This Article aims to examine the contribution of planning, in its dimension of consolidation to the development of Region three of Ecuador during the last decade, which allows understanding the nature of the advances and / or critical nodes in the achievement of development through who have faced this public policy in the region. Methodology: A closed, multiple-choice survey was applied to representatives among public servants, personnel working in universities, private associations; the research work presented used the bibliographic method. Results: The present investigation allows to point out some development indicators of Zone 3 of Ecuador, thus, access to water (irrigation and drinking), health and education in $42.04 \%$ constitutes an important concern for the surveyed citizens, it focuses on agriculture, tourism, services and real estate development that covers $65.19 \%$ of productive activities, and drought, lack of

\footnotetext{
${ }^{1}$ Afiliación: https://orcid.org/0000-0003-0033-1187 (Universidad Católica de Cuenca, Posgrado, Maestría en Desarrollo Local Mención en Ordenamiento Territorial), Cuenca, Ecuador, email (ray.aragon@ucacue.edu.ec) ${ }^{2}$ Afiliación: https://orcid.org/0000-0003-0762-8928 (Universidad Católica de Cuenca, Carrera de economía), Cuenca, Ecuador, email (dorian.flores@ucacue.edu.ec)

${ }^{3}$ Afiliación: https://orcid.org/0000-0002-7710-5199 (Universidad Católica de Cuenca, Posgrado, Maestría en Desarrollo Local Mención en Ordenamiento Territorial), Cuenca, Ecuador, email (ycastilloo@ucacue.edu.ec)
} 
energy, natural disasters and unemployment are the majority risks with $55.46 \%$. Conclusions: In order to have adequate results in the economic and social sphere, through history Ecuador has adopted different economic models that allow the elimination of differences between social classes and economic powers, in order to achieve adequate sustainable development for all sectors and economic activities in the country, that is, there are fewer inequalities.

Keywords: Territorial planning, territorial development, land use planning, planning models, Zone 3 planning

\section{Resumen.}

Introducción: En el marco de estos modelos previos y de la experiencia adquirida, el Ecuador promovió a partir del 2008, la implementación de la planificación como un sistema, basado en un régimen de desarrollo, que determinó planes de ordenamiento y desarrollo territorial por niveles de gobierno, tendientes a la resolución de desequilibrios territoriales que procuren el desarrollo y la equidad socio-económico-ambiental de las poblaciones. Objetivo: El presente Artículo pretende examinar la contribución de la planificación, en su dimensión de consolidación al desarrollo de la Región tres del Ecuador durante la última década, que permita comprender la naturaleza de los avances y/o nudos críticos en la consecución del desarrollo a los cuales se ha enfrentado esta política pública en la región. Metodología: Se aplicó una encuesta cerrada, de opción múltiple, a representantes entre servidores públicos, personal que labora en universidades, asociaciones privadas; el trabajo de investigación presentado empleó el método bibliográfico. Resultados: La presente investigación permite señalar algunos indicadores de desarrollo de la Zona 3 del Ecuador, así, el acceso al agua (de riego y potable), la salud y educación en un 42,04\% constituye una preocupación importante para los ciudadanos encuestados, se enfoca en la agricultura, turismo, servicios y desarrollo inmobiliario que abarca un $65.19 \%$ de las actividades productivas, y la sequía, falta de energía, catástrofes naturales y desempleo son los riesgos mayoritarios con un 55,46\%. Conclusiones: Para disponer de resultados adecuados en el ámbito económico y social, a través de la historia el Ecuador ha adoptado distintos modelos económicos que permitan la eliminación de las diferencias entre las clases sociales y poderíos económicos, a fin de lograr un desarrollo sostenible adecuado para todos los sectores y actividades económicas del país, es decir, que existan menos desigualdades.

Palabras Claves: Planificación territorial, desarrollo territorial, ordenamiento territorial, modelos de planificación, Planificación zona 3. 


\section{Introducción}

El Régimen de Desarrollo en la Constitución del Ecuador (2008) presenta los fundamentos para realizar cambios al modelo de desarrollo, que estaba vigente 20 años antes de esta reforma constitucional en el país, época caracterizada fundamentalmente por la aplicación políticas impulsadas a nivel mundial en la vida económica de América Latina (Martínez, R $\&$ Reyes, G, 2012) que enfatizaban un fortalecimiento económico de la región como el fin último de la política económica, sosteniedo la premisa que el mercado como tal, sería el encargado de la distribución adecuada de la riqueza.

Para ello se promovió la reducción del Estado y la implementación de políticas para la flexibilización laboral y de privatizaciones, que dejaron de lado la inversión social por considerarlo un "gasto", siendo desde entonces este modelo, la plataforma para el impulso del mentado "ajuste estructural" para cumplir en muchos de los casos con las cartas de intención de instituciones mundiales de apoyo y control como como el Banco Interamericano de Desarrollo, Banco Mundial, Fondo Monetario Internacional (Jácome, 2007) principalmente, que tuvo como resultado el incremento en los niveles de desigualdad y pobreza así como de la deuda social en el país.

La Constitución de 2008, en su Título VI presenta el Régimen de Desarrollo en el que se plantea el modelo del "Buen Vivir", con el cual el estado ecuatoriano, mediante un importante sistema de planificación pretende superar las limitaciones del modelo neoliberal anterior.

En ese sentido, la constitución ecuatoriana, en su artículo 275, señala puntualmente: "El régimen de desarrollo es el conjunto organizado, sostenible y dinámico de los sistemas económicos, políticos, socio-culturales y ambientales, que garantizan la realización del buen vivir, del sumak kawsay", así mismo en su art. 276, presenta el enfoque determinado en el Régimen de Desarrollo que proponen la Asamblea (Asamblea Constituyente, 2008), debiendo destacarse entre ellos el control social aunado al fomento de la participación ciudadana que reconoce la diversidad de identidad y la intencionalidad de propiciar una equidad en la representación, como actores activos de la gestión pública con la finalidad de elevar la esperanza y calidad de vida, aumentando así las potencialidades y capacidades de los ciudadanos de la zona, haciendo respetar los derechos establecidos en la Constitución ecuatoriana.

Cabe mencionar que a pesar de que la planificación del desarrollo en el Ecuador se ha venido ejecutando durante varias décadas, el interés que suscita este tema presenta antecedentes importantes en la última década. Es así que el proceso de zonificación nacional surge a partir de la reforma política de 2007 - 2009, plasmado en la Constitución del 2008, que orienta su objetivo, entre otros aspectos, a la desconcentración del accionar público como un 
mecanismo que procure la eficiencia administrativa, al impulso del desarrollo nacional y local, y para acortar las distancias típicamente existentes entre los gobernados y sus gobernantes, distancia mucho más notable en el ámbito de la acción pública estatal.

A partir de ello, se impulsaron varias acciones como la creación de nueva institucionalidad, la expedición de normativas y la división del país en siete zonas de planificación. Este tipo de planificación se ejecuta a partir de diversas instrumentos y herramientas que guían la distribución presupuestaria, la priorización de inversiones públicas, la ejecución y coordinación de competencias, procesos de cooperación internacional, que permitan un accionar estatal desconcentrado.

Esta concepción de un nuevo estado, enfatiza las estructuras regionales desconcentradas, que no obstante presentan varios desafíos, entre los que se pueden mencionar: a) La atención de necesidades específicas de los distintos territorios desde la política pública; b) El Ordenamiento de la ocupación y el uso de los territorios; c) La necesidad de generar y aplicar dinámicas que aporten a la concreción del Pan Nacional de Desarrollo (PND); d) El fomento del desarrollo endógeno de cada región; e) El impulso de una neo-estructura que permita la participación de instituciones públicas de la región, como los más relevantes.

Resulta importante mencionar que, a nivel regional, tanto el dar un nuevo y renovado valor a la planificación del desarrollo como la creación y ejecución de políticas innovadoras enfocadas en el ordenamiento territorial, buscan reducir conflictos prevalentes sobre la ocupación del suelo y el uso que se le da a éste, además de revertir inequidades estructurales, corrigiendo así los desequilibrios territoriales existentes que afectan en gran medida a la competitividad y a la cohesión. Así mismo, el reordenamiento y la propuesta para una descentralización administrativa, política, y fiscal fue implementada para promover tanto el desarrollo local como la democratización, la justicia social y la justicia territorial.

En este marco, este trabajo de investigación examina la planificación -como un instrumento de consolidación-, del desarrollo de la región tres del Ecuador y servirá de base para evaluar y diagnosticar los problemas de desarrollo que confronta la misma. Este territorio, conformado por Tungurahua, Cotopaxi, Pastaza y Chimborazo, fue escogido por los investigadores ya que raras veces, estas provincias son tomadas en consideración para generar estrategias de desarrollo a nivel regional o local.

\section{Marco teórico}

Los autores no se han puesto de acuerdo sobre lo que se considera "desarrollo", éste ha sido abordado desde distintos enfoques, que sin embargo y de manera general, en primera instancia se puede entender al desarrollo como un debate que se amplía, que involucra e 
incluye a sistemas más complejos que afectan a un conjunto de aspectos, como lo es por ejemplo el desarrollo humano de una nación, con todos los componentes que esto implica.

Se debe mencionar que a pesar de estos distintos enfoques su objetivo último se orienta a la búsqueda de políticas en beneficio de las poblaciones, que se puede apreciar en los modelos que se promovieron en las diferentes épocas bajo sus distintos énfasis.

\section{Modelos de desarrollo en América Latina.}

Se refieren al mecanismo aplicado por la sociedad por el cual organiza y gestiona sus recursos, y gestiona sus instituciones con el objetivo de buscar progreso que permita satisfacer adecuadamente los requerimientos de todos los integrantes de una sociedad considerando la diversidad y valorando la capacidad y complejidad de cada grupo social y sus individuos para procurar brindar mejores alternativas de crecimiento social . (Agudelo, 1999).

De acuerdo a Franco (1996) se destacan tres modelos que se emplearon en Latinoamérica durante el siglo 20 y que corresponden: a) Modelo de crecimiento hacia afuera; b) Por sustitución de importaciones; y, c) Modelo de post-ajuste. Por su parte, otros autores reconocen una lista algo más extensa de modelos de desarrollo empleados en América Latina, los mismos que se explican brevemente a continuación. Cabe señalar, que, si bien en la región no se desarrolló lo suficiente el Estado de Bienestar, éste se explica en parte, debido al debate del desarrollo en América Latina a partir del análisis entre dicho modelo y el modelo Neoliberal, éste último predominante en la historia cercana de nuestros países.

\section{Modelo Keynesiano y Estado de Bienestar.}

El término (que en inglés es Welfare-State), hace referencia a la sociedad desarrollada y de consumo. Los inicios de esta línea político-económica son el programa New Deal, promovida por F.D. Roosvelt (expresidente de los Estados Unidos), a partir del año de 1933 con el objetivo de superar la crisis provocada por la Gran Depresión que se dio en ese país por la caída de la bolsa de valores de 1929 en Nueva York (crisis que fue denominada también como crack del 29). Está basado en la teoría de la ocupación y reconoce que las tasas de ocupación, que es el "cociente entre el número de personas ocupadas comprendidas en el rango de edad desde los 16 años hasta los 64 años y la población total que comprende el mismo rango de edad (Población en edad de trabajar (PET))" son indicadores adecuados que reflejan la prosperidad de los países, y que por otra partes, los bajos ingresos o la falta de éstos compromete en gran medida tanto el desarrollo humano como la conservación de la vida (Musgrove, 1993). 
La forma adecuada en la que una nación refleja el bienestar de sus ciudadanos se presenta en la forma de un Estado Social que, mediante la aplicación de políticas y leyes adecuadas, garantiza ciertos estándares en temas como la salud, la alimentación, nivel promedio de ingresos, acceso a vivienda, seguridad social y educación a todos los ciudadanos, sin que exista ningún tipo de discriminación. Este tipo de Estado emergió en resultado a un impulso de estructuras sociales capitalistas y de crecimiento industrial. (Fleury \& Molina, 2002). A partir de sus concepciones, se persigue conseguir un entorno estable y seguro, donde prime la solidaridad social.

\section{Modelo de la Sustitución de importaciones.}

Este modelo generó muchas buenas expectativas con respecto a la promesa de progreso y modernización de los países latinos, sus efectos estaban enmarcados en un aumento tanto en las tasas de crecimiento como en las oportunidades para obtener un trabajo, permitiendo que muchos superen sus niveles de pobreza. A pesar de ello, este modelo mostró debilidades en los años 70's, momento en que la región perdió su competitividad con el resto del mundo, hecho que generó una baja en las oportunidades de exportación acompañada por la elevación de los índices de inflación, estas situaciones desencadenaron la consecuente y famosa "crisis de la deuda" en los años 80, crisis que se caracterizó por altos niveles de desempleo, reducción de sueldos, aumento de la pobreza, incremento de la desigualdad y la tendencia descendente de índices de crecimiento (Rey de Marulanda, 2000).

\section{Modelo Neoliberal.}

Bajo este modelo, los ciudadanos se perciben como clientes, que interactúan en amplios y variados mercados de bienes, servicios y valores sociales, siendo estos artículos mercantiles de vendedores del sector privado cuyo objetivo principal interés la obtención de ganancias económicas. En este modelo, se fomenta y propicia la libre competencia, es justamente el cliente o consumidor quien elige los productos y servicios que consume según su capacidad de compra. Este modelo se caracteriza por el individualismo, ya que en esta visión, el individuo liberal se considera como una isla que se fundamenta y se sostiene gracias a sí misma, enfocándose en el cumplimiento de los derechos a la vida, derecho a la propiedad y derecho a la libertad (Musgrove, 1993).

En América Latina, el modelo neoliberal tuvo su expansión en la década de los 80 de la mano con la aplicación de reformas económicas propuestas por organismos internacionales usadas como métodos para estabilizar las economías en esta región y superar el déficit público (Consenso de Washington) (Albala-Bertrand, 1991). 


\section{Modelo Económico de Planificación Central.}

Usado en América Latina por iniciativas socialistas (como en Cuba y Nicaragua) (Cardona Osorio, 1987). Este modelo fue creado y empleado principalmente en la antigua Unión Soviética, China y Europa Occidental. Se caracteriza porque en éste, el Estado que es el dueño de los recursos es el que decide cómo asignar dichos recursos, organizando también la producción y el tipo de industrias. A inicios del siglo XX se registraron bajo este modelo logros significativos en aspectos como el ambiental, la seguridad, progreso social, registrándose un aumento en la industrialización y producción, garantizando la gratuidad de servicios de salud y educación, empleo y vivienda para los ciudadanos, con la ya conocida distribución equitativa de los ingresos y los servicios sociales que este tipo de modelo propone. En los años 70 inició en la región un proceso de paralización que obligó a los estados que lo utilizaban a emprender reformas sobre todo en lo referente a políticas económicas de mercado (Banco Mundial, 1996). Luego de su debacle, se han planteado alternativas de desarrollo.

El modelo de desarrollo presentado en la Constitución de la República (Asamblea Constituyente, 2008), considera necesario la búsqueda de nuevos mecanismos orientados al respeto de la libertad de decisiones, a un distribución equitativa y eficiente, que tengan un impacto favorable en cuanto a los beneficios que reporten en comparación a los modelos previos. Esta propuesta surgió frente a las consecuencias negativas provocadas por los lineamientos de los modelos de desarrollo anteriores, sobre todo, en cuanto a la distribución inequitativa de la riqueza del país que se presentaban con las habituales políticas centradas en crecimiento del producto, con las cuales la inversión del estado se destinaba a ciertos sectores "estratégicos", hecho que resultaba ineficiente, socialmente inequitativo, injusto, ecológicamente insostenible y económicamente inconsistente. Según este sistema, el estado regula y complementa al mercado, pues, al disponer de mercados eficientes las economías tendrán mayores y mejores resultados en crecimiento y equidad.

Sin embargo, a pesar de que la inversión sea necesaria, en especial de capital extranjero, éstos no siempre garantizarán el crecimiento, al menos no en las tasas que se espera. Esto ha causado que las concepciones modernas de desarrollo se centren en la supresión de distorsiones ocurridas por el estado y la disminución de inconvenientes que resultan al realizar cambios institucionales y sobre todo, de las organizaciones económicas (Meier \& Stiglitz, 2001) del acceso a las nuevas tecnologías para ser parte de la denominada sociedad del conocimiento. En este tipo de modelos es donde más se pueden encontrar las denominadas fallas de gobierno, en las que se presentan, por ejemplo, costos de las transacciones generados cuando existe una intervención del Estado, la retención de ganancias derivadas por procesos de regulación, captura del regulador por partes de grupos privados, administraciones en busca de satisfacer intereses particulares, desencadenando en la toma del 
estado por la corrupción, hecho que ha estado presente desde hace ya varios años en los gobiernos de Latino América.

La concepción actual de desarrollo considera no solamente al crecimiento económico, sino además se prioriza aspectos de garantía de bienestar social, el fomento de la participación social, la garantía del derecho a la libertad, al desarrollo humano y la búsqueda de la democracia. En este sentido, existe una interacción entre los modelos analizados; unos que se basan en principios de mercado propiciando la inversión privada, cuyo propósito es buscar la eficiencia disminuyendo la intervención del Estado. Otros modelos se enfocan en la dotación de recursos y acatar disposiciones del estado, dando mayor importancia al cumplimiento de las funciones sociales del estado, es decir, los esfuerzos por superar la pobreza, mejoramiento de los sistemas educativos, de vivienda, salud, saneamiento básico, entre otros.

En este esquema es importante también la consideración de objetivos pertinentes al logro de estándares de democracia, libertad, derechos humanos, protección ambiental y desarrollo sostenible (Stiglitz, 1998), citado por (Appiah et al., 2020).

Las tendencias de desarrollo actuales se basan en cuatro ejes principales: las teorías de la información asimétrica, el neoinstitucionalismo, las teorías del crecimiento endógeno y la nueva economía política.

Akerlof, Spence y Stiglitz, en la teoría de información asimétrica, señalan como característica fundamental de una economía de mercado es la asimetría de información que poseen entidades económicas (agentes económicos), siendo este un factor decisivo para el crecimiento, para los ciclos económicos y para las políticas públicas.

El neoinstitucionalismo, se centra en el hecho de que las reglas de juego para los ciudadanos productivos de una sociedad son importantes para dar una explicación sobre el desempeño económico, mientras que el cambio institucional revela y determina el por qué algunos Estados presentan un mayor desarrollo que otros. Un importante exponente de esta corriente es North en los años 1990.

Las teorías del crecimiento endógeno, desarrolladas al final de la década de los 80`s por autores como Romero, Lucas y Barro, demuestran la importancia del papel del capital humano y la calidad de este, para la mejora de la productividad y un crecimiento económico, así como en la generación de conocimientos como aspecto decisivo para el desarrollo.

La nueva economía política considera que la economía está atada al entorno social, como parte intrínseca del desarrollo utilizando indicadores e instrumentos utilizados en teorías 
económicas, en un proceso de participación directa del estado y políticas públicas.(Saiegh \& Tommasi, 1998), citado por (Carbajales \& Carbajales, 2019).

En nuestro país hace algunas décadas se han realizado varios esfuerzos para mejorar la planificación y el ordenamiento territorial, esfuerzos que se han constituido en acciones aisladas que no han logrado completamente los objetivos para los que fueron implementados. Es a partir del año 2008, se propone un modelo, denominado plan nacional para el Buen Vivir (año en que el tema del ordenamiento territorial se trata como un asunto constitucional cuyo objetivo es brindar soluciones a las problemáticas de pobreza e inequidad que imperaban en el Ecuador), que se han incorporado ejercicios de planificación a nivel regional y nacional que han rendido frutos importantes. (Lira Cossio, 2006), citado por (Carrozza \& Brieva, 2020) (Selee, 2004), citado por (Vargas-Hernández, 2020).

Uno de ellos fue la definición de zonas administrativas, descritas a continuación por parte del poder ejecutivo (SENPLADES, s/f) mediante la reforma política 2007-2009, con la finalidad de desconcentrar la gestión pública y administrativa según se indica en (Ponce \& Pérez, 2017):

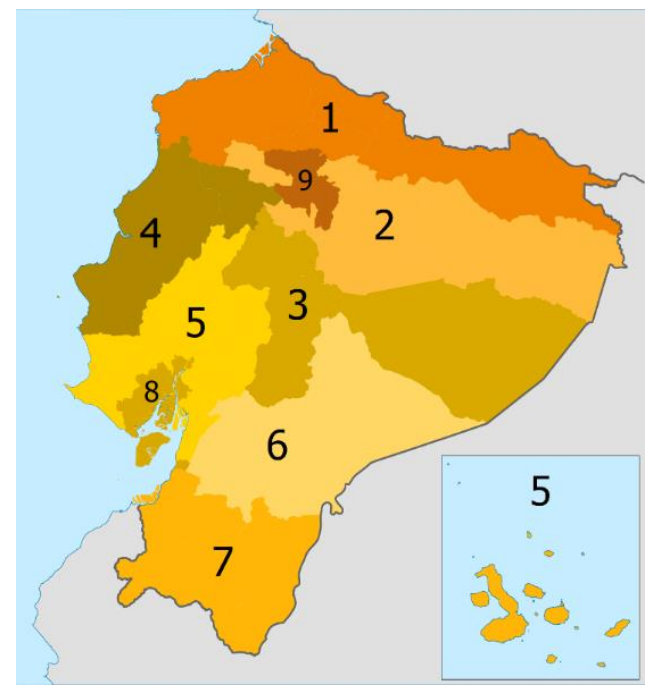

Figura 1: Zonas de planificación del Ecuador

\section{Elaborado por: Los autores}

- Zona 1: Esmeraldas, Imbabura, Carchi, Sucumbíos

- Zona 2: Pichincha, Napo, Orellana

- Zona 3: Cotopaxi, Tungurahua, Chimborazo, Pastaza

- Zona 4: Manabí y Santo Domingo de los Tsáchilas

- Zona 5: Guayas, Santa Elena, Los Ríos, Bolívar y Galápagos 
- Zona 6: Azuay, Cañar y Morona Santiago

- Zona 7: Loja, El Oro y Zamora Chinchipe

- Zona 8: Guayaquil (DM)

- Zona 9: Quito (DM)

En la Ley Orgánica de Ordenamiento Territorial, Uso y Gestión de Suelo (2016), se establece los principios y reglas generales que orientan y disponen las competencias de ordenamiento territorial, y se dispone el ejercicio de uso y gestión del suelo urbano o rural, con el objetivo de propiciar un desarrollo sostenible del territorio, motivando la aplicabilidad del derecho a una vivienda digna, hábitat seguro, e impulsa el desarrollo urbano inclusivo e integrador para el buen vivir de los ciudadanos ecuatorianos (Asamblea Nacional, 2016).

Entre los fines de esta ley, se pueden mencionar la orientación de las políticas públicas en relación al ordenamiento territorial, el establecimiento de mecanismos que orienten el cumplimento y aplicación de las competencias de uso y gestión del suelo de los Gobiernos Autónomos Descentralizados municipales y metropolitanos y del Estado en general; la definición de estrategias para garantizar el uso de suelos con acceso a vivienda adecuada; establecimiento de indicadores mínimos de calidad urbana considerando aspectos de uso de espacios públicos, infraestructuras y servicios básicos; Establecer mecanismos de garantía de soberanía alimentaria y aprovechamiento de recurso.

De este importante documento se desprenden también principios y directrices para el ordenamiento territorial y uso eficiente del suelo, mismo que se presentan en la tabla 1.

Tabla 1: Principios Rectores del Ordenamiento Territorial

Principio

Descripción

Sustentabilidad

La leyes y métodos que dirigen el ordenamiento territorial, gestión y uso del suelo promoverán el manejo eficiente y equitativo de los recursos, el desarrollo sustentable, y la mejora de la calidad de vida tanto de los ciudadanos de hoy como de las futuras generaciones.

Equidad territorial y justicia social
Todas las decisiones sobre temas de territorio se adopten, garantizarán a la ciudadanía que habiten en él, el acceso racional a los servicios básicos y las mismas oportunidades para aprovechar las iniciativas de desarrollo emprendidas, garantizando así el Buen Vivir. 
Autonomía

Coherencia

Concordancia

El derecho a la ciudad
Los GAD municipales dentro de sus funciones, ejercerán sus competencias en cuanto al ordenamiento territorial, uso y gestión del suelo respetando las normas que para este fin se establecen en la Constitución de la República, considerando además de las otras regulaciones nacionales que se emitan en este sentido.

Las decisiones que se tomen con respecto al desarrollo y el ordenamiento territorial deben presentar un alto grado de coherencia con la realidad social de las regiones donde se ejecutarán, para lograr dicha coherencia se deben considerar los aspectos culturales, económicos, ambientales, entre otros, que sean propios de cada territorio y sus individuos.

Las decisiones que sobre los territorios que se tomen a nivel de los GAD’s y los regímenes especiales, deben estar adecuadamente articuladas entre sí, guardando también correspondencia con las disposiciones del nivel jerárquico superior y respetar los anhelados y entes mencionados principios de equidad, integración, solidaridad y participación ciudadana, practicas comunes de la gestión establecidas en los Art. 238 y 260 de la Constitución.

a) Se debe garantizar el ejercicio de la ciudadanía, mismo que asegurará el bienestar y la dignidad colectivos de los pobladores de una región, bajo las necesarias condiciones de justicia, equidad e igualdad.

b) La gestión de las urbes debe realizarse de manera democrática, es decir mediante la aplicación de acciones directas que permitan la participación democrática de los habitantes de las ciudades en la gestión y planificación de las mismas, así como con la utilización de estrategias de información, rendición de cuentas y transparencia para la ciudadanía.

c) En cuanto a la función ambiental y social de la propiedad, es menester que se priorice el interés general sobre el interés 
Principio

\section{Descripción}

particular. Así mismo, se debe garantizar el derecho a un hábitat (vivienda) que sea seguro y saludable para los miembros de la sociedad.

La función pública del urbanismo

La distribución equitativa de las cargas y los beneficios
Las decisiones que se adopten sobre planificación y gestión del territorio deberán priorizar el interés público, ponderando las necesidades reales del contexto de los ciudadanos que habitan la urbe, garantizando de esta forma el derecho de los habitantes a vivir en una vivienda digna, en un entorno seguro y saludable, con servicios básicos y con espacios públicos adecuados y de calidad.

Mediante las acciones que se emprendan, se debe garantizar el justo y equitativo reparto de las obligaciones y derechos entre todos los actores que intervienen en los procesos urbanísticos, esto debe realizarse conforme lo establen las normas que reglamentan dichos procesos y dentro del marco de una adecuada planificación.

El Plan Nacional de Desarrollo se orienta en la premisa principal del cumplimiento de los objetivos nacionales establecidos, enfocados un mejoramiento de la calidad de vida, por medio de políticas públicas que generen proyectos implementados en territorio. La Agenda de la Coordinación Zonal 3 es elaborada justamente basado en un análisis íntegro de las provincias de Pastaza, Chimborazo, Cotopaxi y Tungurahua, profundizando en temas de entorno físico, requerimientos insatisfechas, procesos propios de planificación, articulación organizada entre gobiernos descentralizados y entidades desconcentradas para la aplicación de las políticas públicas establecidas. El documento trata sobre un análisis de situación de la Zona 3 en la actualidad, con la información de la percepción del ciudadano en este ámbito, que se detalla a continuación:

Problemas sociales: a) Bajo nivel de acceso a agua segura, educación, recolección de desechos, alcantarillado, salud, b) Altos índices de pobreza, c) Riesgos de soberanía alimentaria, d) Desestructuración de familias por la migración, e) Bajo acceso a casa propia y digna. 
Problemas Productivos: a) Niveles de productividad bajos, b) Falta de una adecuada planificación en cuanto a la producción agropecuaria, c) Producción sin encadenamientos productivos y de valor, d) Limitado o ningún acceso a recursos importantes para la producción (tierra, capital, agua, tecnología y conocimiento), e) Elevación de los costos de producción, f) falta de normativas que exijan mayores estándares de calidad, g) Escases de leyes que faciliten y posibiliten la utilización y ocupación del suelo de acuerdo a su aptitud productiva, h) Escasos mecanismos que permitan fortalecer la producción a escala nacional.

Problemas Ambientales: a) Acelerada disminución de los glaciares en los volcanes de la región, b) Cambio en los habituales períodos de lluvias, c) Disminución de agua (calidad y calidad en cursos naturales y artificiales), d) Disminución de la superficie de bosques de montañas, bosques y páramos, e) Alta erosión de los suelos, f) Conflictos causados por el uso de suelo.

Y se analizan los problemas habituales que se encuentran en la planificación de la región y que se detallan más adelante.

Para ello, se utilizó la aplicación de un método con enfoque mixto, por un lado, es longitudinal y se desarrolló en un escenario que involucró la participación de 384 personas que habitan en la zona 3 del Ecuador (provincias de Chimborazo, Cotopaxi, Pastaza y Tungurahua). Los investigadores desean conocer la percepción de dichos ciudadanos con respecto a los problemas detectados sobre temas de ordenamiento territorial; la muestra con la que se trabajó fue obtenida empleando muestro estratificado proporcional, no probabilístico y discrecional. Los investigadores enviaron el enlace de una encuesta virtual realizada en Google Form.

La zona 3, proyectada al año 2020, tiene aproximadamente 1.677.761 de habitantes, cantidad que representa al $9.86 \%$ de la población total del Ecuador, distribuida por provincia de la siguiente manera: Cotopaxi con 476.428; Tungurahua con 577.551; Chimborazo con 515.417 y Pastaza con 108.365 habitantes, aplicando la fórmula correspondiente se obtiene que la muestra es de 88 sujetos, en la cual y por el criterio de estratificación proporcional, Chimborazo representa y aporta con un $30.72 \%$ a la muestra, Cotopaxi con $28.39 \%$, Pastaza con $6.45 \%$ y Tungurahua con $34.42 \%$.

El trabajo de investigación presentado empleó el método bibliográfico, el cual consistió en la revisión de bibliografía publicada en bases de datos científicas especializadas, en busca de información pertinente, válida y relevante sobre los temas de desarrollo, planificación y organización territorial, la misma que derivó en aspectos que permitieron determinar las problemáticas comunes en relación con la organización y planificación territorial en la zona 3 del país. Se empleó además el método descriptivo, con el que se detallaron los conceptos y 
las teorías más importantes sobre las que se sustentan tanto las tendencias de desarrollo (y sus pilares) como los procesos.

\section{Resultados}

La investigación pretende brindar con una panorámica de desarrollo territorial en la zona 3 del Ecuador, una propuesta de modelo adecuado de desarrollo y ordenamiento territorial a proponer, basado en la aplicación de encuestas, para la identificación de las temáticas fundamentales regionales para la construcción de dicha visión. En ese contexto, los investigadores han priorizado y seleccionado las siguientes dimensiones:

- Temáticas regionales importantes

- Actividades productivas y Económicas

- Amenazas o aspectos vulnerables

- Proyectos, áreas, temáticas e iniciativas

- Metas relevantes pasa loa zona 3 (Ecuador)

Para la obtención de los resultados se elaboró una encuesta cerrada, en la que se debían seleccionar una opción para cada uno de los indicadores en que se constituyó la encuesta aplicada. La encuesta se envió y difundió en cada provincia de la zona 3 por correo electrónico el 5 de octubre de 2020 estableciéndose un plazo de dos semanas para la recepción de respuestas (hasta el 19 de octubre de 2020). Se enviaron un total de 100 encuestas en cada provincia, a un conjunto de personas entre los que constaban: servidores públicos, personal que labora en universidades, asociaciones privadas intentando abarcar de manera equitativa a instituciones públicas y privadas.

De entre todas las respuestas que se obtuvieron al aplicar la encuesta (Chimborazo 64; Cotopaxi 73; Pastaza 55; Tungurahua 62), se procedió a obtener el número de sujetos de estudio aplicando para ello muestreo aleatorio simple (MAS) para obtener el número de participantes de cada provincia. Con los resultados se realizó un análisis de cómo, se aporta a una visión de Desarrollo Regional. Se presentan a continuación los resultados de este análisis.

\section{1.- Priorización de temáticas Regionales relevantes para el correcto desarrollo de la Zona tres del Ecuador.}

Temáticas según su importancia

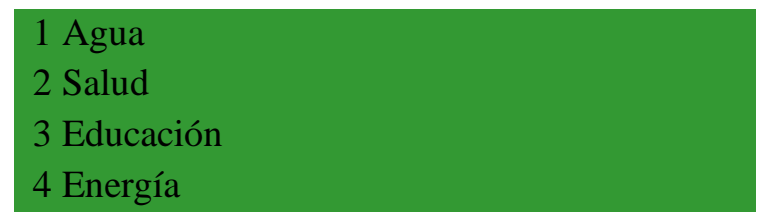

\section{Porcentaje en función de la frecuencia}

Agua 19,32\%

Salud $11,36 \%$

Educación 11,36\%

Transporte, viabilidad... 7,95\% 


\section{Temáticas según su importancia}

5 Empleo
6 Transporte, vialidad y conectividad
7 Seguridad
8 Descentralización
9 Minería
10 Patrimonio, cultura e identidad
11 Innovación
12 Integración internacional
13 Vivienda
14 Crecimiento de centros poblados
15 Amenazas naturales
16 Espacio público
17 Cohesión social
18 Biodiversidad

\section{Porcentaje en función de la frecuencia}

Empleo 7,95\%

Energía 7,95\%

Seguridad ciudadana $5,68 \%$

Descentralización $4,55 \%$

Minería 3,41\%

Vivienda $3,41 \%$

Patrimonio, cultura... 3,41\%

Innovación 3,41\%

Crecimiento de centros... 2,27\%

Integración internacional 2,27\%

Amenazas naturales 2,27\%

Espacio público 1,36\%

Cohesión social 1,36\%

Biodiversidad 1,36\%

Como se aprecia en los resultados de la pregunta 1, el agua tiene mayor prioridad en cuanto a las temáticas consideradas importantes para el desarrollo territorial, hecho que apunta a una revisión de los lineamientos pertinentes orientados a la optimización y eficiencia en el manejo de recursos hídricos. Resulta importante señalar que los temas referentes a transporte, vialidad y conectividad y energía resultaron ser relevantes dentro de la priorización, hecho que destaca su importancia para el desarrollo regional, este rol importante hace necesaria la ejecución de estudios de potenciales generadores energéticos, así mismo se requerirán estudios que mejoren la red vial de la zona.

\section{2.- Actividades productivas y económicas en el desarrollo zonal, según su importancia.}

\section{Actividades según su importancia}

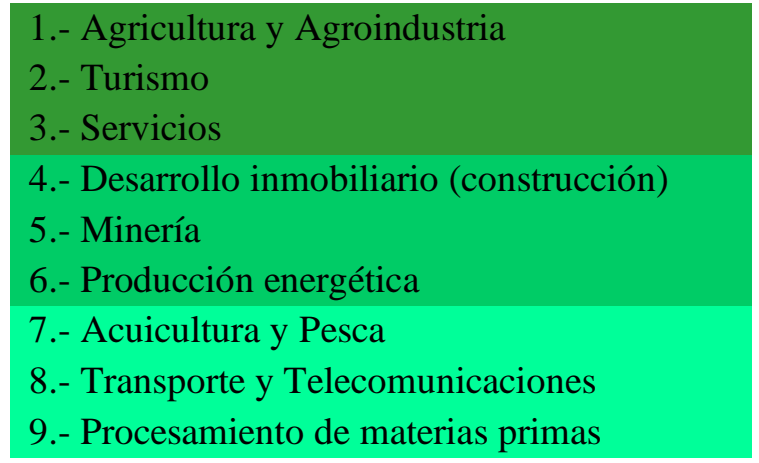

\section{Porcentaje en función de la frecuencia}

Agricultura y agroindustria $19,32 \%$
Turismo $18,18 \%$
Servicios $17,05 \%$
Desarrollo inmobiliario... 13,64\%
Minería $11,36 \%$
Acuicultura y Pesca $9,09 \%$
Producción energética $6,82 \%$
Transporte y Telecomunicaciones 3,41\%
Procesamiento de materias primas 1,14\%

Al analizar los resultados de la pregunta 2, se puede apreciar que los temas referentes a turismo, Agricultura y Servicios lideran las actividades económicas y productivas, hecho relacionado con las áreas que presentan una mayor representación en el Producto Interno 
Bruto de la zona, de igual forma, reconocer la importancia de los Servicios y el Desarrollo inmobiliario (construcción), hacen notar la importancia que tiene la región como destino atractivo para migrar y como una plataforma de servicios.

Resulta importante destacar que Producción energética (que sobrepasa a Transporte y Telecomunicaciones y Procesamiento de materias primas), da cuenta de los esfuerzos realizados en la zona en cuanto a energía y al potencial que este tema presenta como actividad económica, con iniciativas que podrían implantarse como la de generación de energía eólica, entre otras.

\section{3.- Amenazas y riesgos para el desarrollo de la zona 3 del Ecuador.}

\section{Ítems según su importancia}

1.- Sequia
2.- Falta de energía (para sectores
productivos)
3.- Desempleo
4.- Catástrofes naturales
5.- Variaciones del mercado mundial
6.- Migración campo - ciudad
7.- Alteraciones en la economía nacional
8.- Contaminación antrópica (causada por el
hombre)

\section{Porcentaje en función de la frecuencia}

Sequia $25,00 \%$

Falta de energía $17,05 \%$

Catástrofes naturales $13,64 \%$

Desempleo $11,36 \%$

Variaciones del Mercado Mundial 10,23\%

Migración campo - ciudad 7,95\%

Alteraciones en la economía nacional 7,95\%

Contaminación antrópica $6,82 \%$

En cuanto a los resultados de la pregunta 3, es importante mencionar que la sequía lidera la priorización, haciendo notar la preocupación de las personas de la zona ante este hecho y las consecuencias que tendría en otras áreas del desarrollo, esto resulta coherente con la priorización del tema agua cuando se hable de desarrollo regional. El tema de la energía también se reporta como una preocupación importante en temas de desarrollo. Claro está, que el desempleo también es un factor importante que amenaza el desarrollo de la zona, al igual que el desarrollo de la mayoría de las provincias del Ecuador.

\section{4.- Proyectos, temáticas, áreas e iniciativas a potenciar en la zona}

\section{Ítems según su importancia}

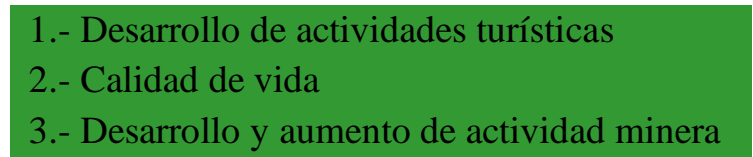

\section{Porcentaje en función de la frecuencia}

Desarrollo de actividades turísticas 20,45\%

Calidad de vida $14,77 \%$ 


\section{Ítems según su importancia}

4.- Desarrollo y aumento de actividad
agrícola
5.- Innovación en tecnología e industria
6.- Fortalecimiento como polo educacional de
la zona
7.- Producción de la matriz energética (y su
diversificación)
8.- Patrimonio, cultura e identidad
9.- Desarrollo y crecimiento de actividades
inmobiliarias

\section{Porcentaje en función de la frecuencia}

Fortalecimiento como polo educac... 12,5\%

Desarrollo de la actividad minera 10,23\%

Desarrollo de la actividad agrícola 10,23\%

Innovación en tecnología e industria 9,09\%

Producción de la matriz energética (y su diversificación) $7,95 \%$

Patrimonio, cultura e identidad 7,95\%

Desarrollo y crecimiento de actividades inmobiliarias $6,82 \%$

En cuanto a los temas que deben potenciarse, tratados en la pregunta 4 de la encuesta, se advierte que la actividad turística se consolida como la más relevante, cabe resaltar que en el punto 2 esta actividad fue se calificó como la segunda más importante para la zona, observándose que esta actividad posee un gran potencial. Cabe destacar también que la calidad de vida se refleja como un tema que debe ser potenciado, siendo este tema una preocupación que se debe mantener en el tiempo y que debe extenderse a toda la zona.

\section{5.- Metas relevantes para la zona 3 al año 2030}

\section{Metas según su importancia}

1.- Crecimiento económico
2.- Disminución de la pobreza
3.- Sistema de salud de calidad
4.- Educación de calidad
5.- Calidad de vida en zonas rurales y urbanas
6.- Transporte, conectividad y vialidad de
calidad
7.- Diversificación económica
8.- Resguardo de territorios (equilibrio
ambiental)
9.- Ciudades seguras

\section{Porcentaje en función de la frecuencia}

Crecimiento económico 19,32\%

Disminución de la pobreza $17,05 \%$

Sistema de salud de calidad $13,64 \%$

Transporte, conectividad y vialidad de calidad $12,5 \%$

Calidad de vida en zonas rurales y... 11,36\%

Educación de calidad $11,36 \%$

Diversificación económica $6,82 \%$

Resguardo de territorios... 4,55\%

Ciudades seguras $3,41 \%$

En cuanto a la pregunta 5, al observar las 4 primeras metas consideradas como más importantes, se nota la existencia de anhelos y preocupaciones transversales a los territorios y habitantes de la zona. A partir de estas metas, las que les siguen se relacionan con el aporte que puede hacerse para la consecución de metas transversales para el desarrollo zonal. 


\section{Conclusiones}

- La zona de planificación 3 (integrada por Tungurahua, Chimborazo, Cotopaxi y Pastaza), por su ubicación en el centro país y su clima privilegiado, posee un gran número de parques nacionales y áreas protegidas que son beneficiosos para el desarrollo de las actividades turísticas, sin dejar de lado el hecho de que la biodiversidad y la riqueza natural de la región también han hecho posible el desarrollo de actividades como la agricultura y la ganadería, la silvicultura, producción de medicamentos naturistas (en base de hierbas), la naturopatía , entre otras. Es necesario señalar que, en ausencia de intervenciones correctamente planificadas para proteger el uso sostenible de los recursos naturales que soportan las actividades antes mencionadas, la zona 3 de planificación puede estar en un proceso de deterioro, en cuyo caso, se dificultaría el desarrollo social y económico de los ciudadanos de la región, poniendo freno al anhelado crecimiento y desarrollo de la zona. En el caso de presentarse este escenario adverso, el nivel de pobreza aumentará, la provisión de servicios e infraestructura básicos será insuficiente, la producción de alimentos disminuirá y el desempleo aumentará, lo que podría provocar en un caso extremo, un aumento de la migración de los habitantes hacia otras zonas del país o al exterior.

- El análisis general del trabajo presentado, detectó que los principales problemas que deben ser revisadas y considerados en el marco de una adecuada planificación para los territorios de la zona 3 de nuestro país son: a) No consideración del deterioro que sufren los Recursos Naturales (RR.NN) de la zona; b) No consideración de la influencia del estado de los RR.NN en el aumento de riesgos; c) Aumento de zonas marginales en las urbes de la zona (debido en muchos casos por la expansión no planificada de las ciudades); d) Las cuestiones referentes a la conectividad, telecomunicaciones, suministro de energía eléctrica, agua y demás servicios, deben ser revisados y priorizados en la planificación; e) Disminución de las zonas agrícolas en la zona (debido en unos casos al abandono de las tierras por parte de los agricultores que migra a otras ciudades o al exterior, y en otros casos por la expansión de las zonas urbanas que emplean suelos que antes se destinaban para la producción agrícola), este hecho ocurre también en el caso de zonas ganaderas, en ambos casos se genera un impacto sobre la soberanía alimenticia de la zona y del resto del país; f) Se deben adoptar acciones que aumenten la precaria capacidad para generar empleo, para mitigar la consecuente concentración de la pobreza en la zona 3 y mejorar las condiciones de vida de sus habitantes.

- La presente investigación permite señalar que en cuanto a la dimensión Temáticas Regionales Relevantes para el correcto desarrollo de la Zona 3 del Ecuador, el acceso al agua (de riego y potable), la salud y educación constituye una preocupación 
importante para los ciudadanos encuestados. En lo que se refiere a las actividades productivas y económicas de la zona, la agricultura y la agroindustria se perciben como los más importantes, seguido por turismo y prestación de servicios. Las sequías, falta de energía y las catástrofes naturales se reportan como las principales amenazas y riesgos que enfrenta la zona 3 para su desarrollo. El cuanto a la dimensión Proyectos, Temáticas, Áreas e Iniciativas que se deben potenciar en la zona 3, los datos arrojados por la encuesta aplicada permiten determinar que el desarrollo de actividades turísticas, calidad de vida y educación se percibe como los aspectos más importante que debería ser impulsado en la zona, en cuanto a las metas consideradas como relevantes se puede indicar que el Crecimiento económico seguido de la Disminución de la pobreza y el anhelo de un Sistema de salud de calidad son las preocupaciones más importantes.

- Esta investigación permite evidenciar que la solución de estos problemas requiere de decisiones políticas que prioricen el bienestar de las personas de la zona 3 de Ecuador, es por eso que el modelo económico que se aplique, debe estar enfocado principalmente al bienestar de los ciudadanos. En este sentido, la estructura económico-productiva no puede, ni debería entenderse como un fin en sí misma, sino que tendría que ser considerado el medio por el cual se impulsen las condiciones que beneficien y brinden bienestar a la comunidad de la zona. En este contexto, este componente debe promover una economía de retorno circular y con enfoque solidario.

- En cuanto al componente cultural, los gobiernos seccionales de la zona deben estar conscientes de la riqueza cultural que poseen las provincias que la conforman. Las culturas indígenas que habitan estos territorios, la herencia ideológica (y cultural) que se creó mediante un histórico y complejo proceso de mestizaje, la migración campo - ciudad (y desde el extranjero) entre otros aspectos, hacen que esta región del país sea una zona muy diversa. Es en este escenario, es importante desarrollar iniciativas que orienten su acción a la educación y la sensibilización -el trabajo con grupos vulnerables incidirá positivamente en este componente-, los gobiernos seccionales de la zona deben estar conscientes del gran esfuerzo que debe realizarse para proteger a personas en situación de vulnerabilidad, y a las personas discriminadas por aspectos raciales, culturales, religiosos, etc.

- Otros aspectos importantes a considerar para conseguir el anhelado desarrollo son los referidos a los asentamientos humanos, la movilidad, la energía y las telecomunicaciones. Por ello, en concordancia con las competencias de los GAD municipales se deben emprender acciones y soluciones que aporten a una mejor distribución de asentamientos humanos, considerando además los riesgos potenciales que se generarían si no se diferencian las zonas cuyo suelo debe ser de uso comercial, industrial, espacios verdes, agrícola, para espacios recreativos, etc. de las zonas aptas 
para habitar. Es importante también la implementación y gestión de infraestructura de servicios básicos, la creación de políticas que consideren los procesos migratorios de la zona, el adecuado manejo de energía eléctrica y la potenciación de la red de telecomunicaciones, misma que hará posible una comunicación de calidad, estos aspectos entre otros son los elementos que deben ser priorizados.

- Con estos antecedentes, por tanto, se podría concluir que dentro de las principales problemáticas y que se presentan como nudos críticos de la planificación de la zona tres del Ecuador, hay varios factores, como son entre los más notables, la inestabilidad y debilidad política, social, económica de la región, logrando que los políticos y técnicos cobren excesiva importancia en cada una de sus provincias, cantones, parroquias. Es también palpable la falta de tecnología y capacitaciones periódicas, que permitan registrar, almacenar, analizar e intercambiar información actualizada con todos los niveles de Gobierno, para poder articularse al Plan Nacional de Desarrollo.

- Entre otros problemas visibles se encuentra el hecho de que las administraciones tanto nacionales como locales, tienen poca experiencia en planificación, que los horizontes de los programas y proyectos son de corto plazo, que existe debilidad en la coordinación y articulación interinstitucional, debido a la carencia de mecanismos e instrumentos que obliguen a intercambiar información y a tomar decisiones acertadas, a lo que hay que añadir la escasez de profesionales debido a la ausencia de estabilidad laboral, y a la alta rotación de los mismos ya que algunos cargos son de libre remoción y los continuos cambios administrativos centrales.

- Como consecuencia de la escasa experiencia en las administraciones tenemos la falta de cooperación y coyunturas nacionales e internacionales, que financien proyectos de desarrollo en la región, que muchas veces tiene gran impacto en las dinámicas territoriales. Finalmente, los escasos recursos económicos obligan a las administraciones locales a concentrarse en resolver problemas urgentes en corto tiempo, logrando así interrumpir y/o modificar la planificación, con proyectos improvisados, que, como consecuencia, impiden el desarrollo sostenible de la zona tres del Ecuador.

- Se concluye que, para disponer de resultados adecuados en el ámbito económico y social, a través de la historia el Ecuador ha adoptado distintos modelos económicos que permitan la eliminación de las diferencias entre las clases sociales y poderíos económicos, a fin de lograr un desarrollo sostenible adecuado para todos los sectores y actividades económicas del país, es decir, que existan menos desigualdades. Sin embargo, los modelos han demostrado que eran planteados sin considerar la participación democrática de todos los sectores, razón por la cual la zona tres del Ecuador no ha alcanzado un adecuado crecimiento y desarrollo sostenible en el cual se priorice a las personas y a la naturaleza como ejes centrales de su accionar. 
- Es así que la zona tres no ha alcanzado aun, el nivel de desarrollo esperado y adicionalmente las medidas adoptadas por el régimen no han logrado una conexión entre crecimiento y desarrollo económico, ni los objetivos propuestos por el sistema de planificación que se recogen en las normativas vigentes.

\section{Referencias bibliográficas.}

Agudelo, C. (1999). Desarrollo y salud. Revista de Salud Pública, 1(1), 17-28.

Albala-Bertrand, J. M. (1991). John Williamson (ed.), Latin American Adjustment. How Much has Happened? (Washington, D.C.: Institute for International Economics, 1990), pp. xv + 445. Journal of Latin American Studies, 23(2), 470-472. https://doi.org/10.1017/S0022216X00014310

Appiah, M., Frowne, D. I., \& Tetteh, D. (2020). Re-examining the Nexus Between Financial Development and Poverty Reduction: Evidence from Emerging Economies. Applied Economics Journal, 27(2), 125-144.

Asamblea Nacional, E. (2016). LEY_ORGÁNICA_DE_ORDENAMIENTO_TERRITORIAL_USO_Y_GE_63.31.

Banco Mundial. (1996). Informe sobre el desarrollo mundial 1996. De la planificación centralizada a la economía de mercado.

Carbajales, M., \& Carbajales, M. (2019). The regulatory State. Implications for the judicial control of administrative discretion. Díkaion Revista de Fundamentación Jurídica, 28(1), 69-89. https://doi.org/10.5294/dika.2019.28.1.3

Cardona Osorio, J. (1987). El concepto de salud, enfermedad y salud publica segun los diferentes modos de produccion in Administracion publica de Salud. Le concept de santé, maladie et santé publique selon les différents modes de production. $E l$ concepto de salud, enfermedad y salud publica segun los diferentes modos de produccion in Administracion publica de Salud. Le concept de santé, maladie et santé publique selon les différents modes de production, 12, 103-136.

Carrozza, T. J., \& Brieva, S. S. (2020). Políticas de CTI en el agro y los procesos de instrumentación: (Re)pensando el papel de la planificación estratégica. http://repositorio.flacsoandes.edu.ec/handle/10469/16352 
Fleury, S., \& Molina, C. (2002). Modelos de protección social. Banco Interamericano de Desarrollo. Diseño y gerencia de políticas y programas sociales., 3-6.

Lira Cossio, L. (2006). Revalorización de la planificación del desarrollo. CEPAL. https://repositorio.cepal.org//handle/11362/7316

Martínez Rangel, R., \& Reyes Garmendia, E. S. (2012). El Consenso de Washington: La instauración de las políticas neoliberales en América Latina. Política y cultura, 37, 35-64.

Meier, G., \& Stiglitz, J. (2001). Frontiers of Development Economics (world). https://elibrary.worldbank.org/doi/pdf/10.1596/0-1952-1592-3

Musgrove, P. (1993). Relaciones entre la salud y el desarrollo. Boletín de la Oficina Sanitaria Panamericana (OSP), 114(2). https://iris.paho.org/bitstream/handle/10665.2/16364/v114n2p115.pdf?sequence=1

Ponce, W., \& Pérez, J. (2017). Modelo de Desarrollo Turístico Sostenible para cantones costeros: Herramienta para actores locales de Manabí, Ecuador. Posgrado y Sociedad. Revista Electrónica del Sistema de Estudios de Posgrado, 15, 65. https://doi.org/10.22458/rpys.v15i2.1965

Rey de Marulanda, N. (2000). América Latina: Pobreza y desigualad durante 50 años de reformas económicas y sociales. Washington: Banco Interamericano de Desarrollo/ Instituto Interamericano para el Desarrollo Social; , 6-7.

Saiegh, S. M., \& Tommasi, M. (1998). La nueva economía política, racionalidades e instituciones. Eudeba, 10, 33.

Secretaría Técnica Planifica Ecuador. (2019). Zona de Planificación 3 - Centro Secretaría Técnica Planifica Ecuador. https://www.planificacion.gob.ec/5799/

Selee, A. (2004). Exploring the link between decentralization and democratic governance. Decentralization and democratic governance in Latin America, 3-36.

SENPLADES. (s/f). La Planificación: UN INSTRUMENTO PARA CONSOLIDAR LA REGIONALIZACIÓN. https://www.planificacion.gob.ec/wpcontent/uploads/downloads/2012/08/La-Planificaci\%c3\%b3n.pdf

Stiglitz, J. (1998). The Role of the Financial System in Development. Presentation at the Fourth Annual Bank Conference on Development in Latin America and the Caribbean, 29, 17. 
Vargas-Hernández, J. G. (2020). Urban Governance, Democratic Decentralization, and Natural Resources [Chapter]. Advanced Integrated Approaches to Environmental Economics and Policy: Emerging Research and Opportunities; IGI Global. https://doi.org/10.4018/978-1-5225-9562-5.ch009 


\section{PARA CITAR EL ARTÍCULO INDEXADO.}

Flores Aguilera, D. D., Aragón León, R. J., \& Castillo Ortega, Y. (2021). La planificación, un instrumento para consolidar el desarrollo local en el caso de la región tres del Ecuador . ConcienciaDigital, 4(1.2), 54-78. https://doi.org/10.33262/concienciadigital.v4i1.2.1580

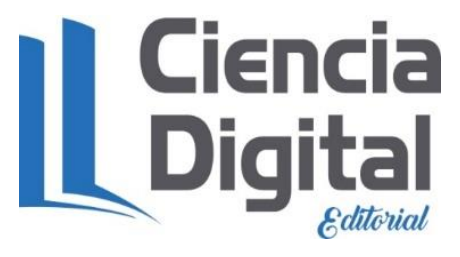

El artículo que se publica es de exclusiva responsabilidad de los autores y no necesariamente reflejan el pensamiento de la Revista Conciencia Digital.

El artículo queda en propiedad de la revista y, por tanto, su publicación parcial y/o total en otro medio tiene que ser autorizado por el director de la Revista Conciencia Digital.
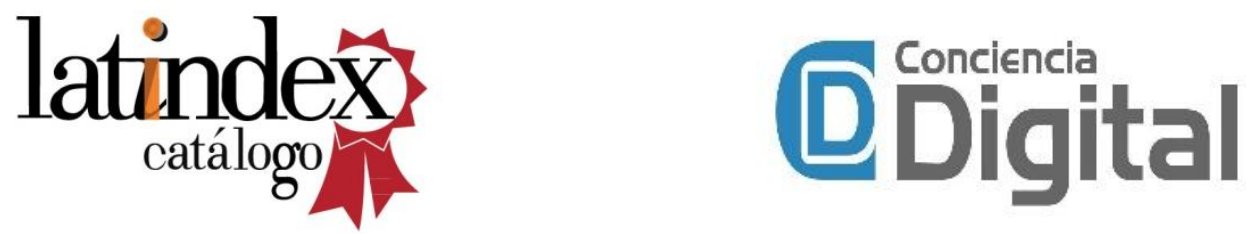